\title{
Some generalized Volterra-Fredholm type dynamical integral inequalities in two independent variables on time scale pairs
}

\author{
Haidong Liu' ${ }^{*}$ (D) and Chuancun Yin²
}

\section{"Correspondence:}

tomlhd983@163.com

${ }^{1}$ School of Mathematical Sciences,

Qufu Normal University, Qufu, China

Full list of author information is

available at the end of the article

\begin{abstract}
In this paper, we study some new Volterra-Fredholm type dynamical integral inequalities in two independent variables on time scale pairs, which provide explicit bounds on unknown functions. These inequalities generalize and extend some known inequalities and can be used as effective tools in the qualitative theory of certain classes of partial dynamic equations on time scales. Finally, an example is provided to illustrate the usefulness of our result.
\end{abstract}

Keywords: Time scale; Dynamical integral inequality; Volterra-Fredholm type; Two independent variables

\section{Introduction}

Beginning in 1988, a seminal paper by Stefan Hilger [1] initiated a theory capable of containing both continuous and discrete analysis in a consistent way. Since then, the theory has attracted wide attention. As one of the most fundamental objects, dynamic equations on time scales has been extensively investigated in recent years, we refer the reader to the books [2,3] and to the papers [4-24] and the references therein.

As we all known, inequalities are a powerful tool in the study of qualitative properties of solutions of differential, integral, and difference equations, and so on. During the last few years, a lot of dynamic inequalities have been extended by many authors. See [25-37]. For example, Anderson [28] considered the following nonlinear integral inequality in two independent variables on time scale pairs:

$$
u^{p}(x, y) \leq a(x, y)+b(x, y) \int_{x_{0}}^{x} \int_{y}^{\infty}\left[c(s, t) u^{q}(s, t)+d(s, t) u^{r}(s, t)+e(s, t)\right] \widetilde{\nabla} t \Delta s
$$

Ferreira and Torres [36] studied the following nonlinear integral inequality in two independent variables on time scale pairs:

$$
u^{p}(x, y) \leq a(x, y)+b(x, y) \int_{x_{0}}^{x} \int_{y_{0}}^{y} c(x, y, s, t) u^{q}(s, t) \Delta t \Delta s .
$$

(c) The Author(s) 2020. This article is licensed under a Creative Commons Attribution 4.0 International License, which permits use, sharing, adaptation, distribution and reproduction in any medium or format, as long as you give appropriate credit to the original author(s) and the source, provide a link to the Creative Commons licence, and indicate if changes were made. The images or other third party material in this article are included in the article's Creative Commons licence, unless indicated otherwise in a credit line to the material. If material is not included in the article's Creative Commons licence and your intended use is not permitted by statutory regulation or exceeds the permitted use, you will need to obtain permission directly from the copyright holder. To view a copy of this licence, visit http://creativecommons.org/licenses/by/4.0/. 
Volterra-Fredholm-type integral inequality is an important integral inequality, which contains a definite integral of the unknown function, and has been given much attention by many authors, see [38-46] and the references therein. For example, Feng et al. [38] studied the following Volterra-Fredholm-type finite difference inequality:

$$
\begin{aligned}
u^{p}(m, n) \leq & a(m, n) \\
& +\sum_{s=m_{0}}^{m-1} \sum_{t=n_{0}}^{n-1}\left[c(s, t, m, n) u^{q}(s, t)+\sum_{\xi=m_{0}}^{s} \sum_{\eta=n_{0}}^{t} d(\xi, \eta, m, n) u^{r}(\xi, \eta)\right] \\
& +\sum_{s=m_{0}}^{M-1} \sum_{t=n_{0}}^{N-1}\left[f(s, t, m, n) u^{l}(s, t)+\sum_{\xi=m_{0}}^{s} \sum_{\eta=n_{0}}^{t} g(\xi, \eta, m, n) u^{k}(\xi, \eta)\right] .
\end{aligned}
$$

Meng and $\mathrm{Gu}$ [46] considered the following nonlinear Volterra-Fredholm-type dynamic integral inequality on time scales:

$$
\begin{aligned}
u(x) \leq & k+\int_{x_{0}}^{x} f_{1}(s) w(u(s)) \Delta s+\int_{x_{0}}^{x} f_{2}(s) \int_{x_{0}}^{s} f_{3}(\tau) w(u(\tau)) \Delta \tau \Delta s \\
& +\int_{x_{0}}^{\alpha} f_{1}(s) w(u(s)) \Delta s+\int_{x_{0}}^{\alpha} f_{2}(s) \int_{x_{0}}^{s} f_{3}(\tau) w(u(\tau)) \Delta \tau \Delta s .
\end{aligned}
$$

But to our knowledge, Volterra-Fredholm-type dynamic integral inequalities in two independent variables on time scale pairs have been paid little attention in the literature so far. Motivated by the work done in $[36,38,46]$, in this paper, we establish some generalized Volterra-Fredholm-type dynamic integral inequalities in two independent variables on time scale pairs, which not only extend some existing results in the literature, unify some known continuous and discrete inequalities, but also may be applied to the analysis of certain classes of partial dynamic equations on time scales.

\section{Preliminaries}

In what follows, we assume that $\mathbf{T}_{1}$ and $\mathbf{T}_{2}$ are two time scales with at least two points, $x_{0}, \alpha \in \mathbf{T}_{1}, y_{0}, \beta \in \widetilde{\mathbf{T}}_{2}, \alpha>x_{0}, \beta>y_{0}, \widetilde{\mathbf{T}}_{1}=\left[x_{0}, \infty\right) \cap \mathbf{T}_{1}, \widetilde{\mathbf{T}}_{2}=\left[y_{0}, \infty\right) \cap \mathbf{T}_{2}, I_{1}=\left[x_{0}, \alpha\right] \cap \mathbf{T}_{1}$, $I_{2}=\left[y_{0}, \beta\right] \cap \mathbf{T}_{2}, D=\left\{(x, y, s, t) \in \widetilde{\mathbf{T}}_{1} \times \widetilde{\mathbf{T}}_{2} \times \widetilde{\mathbf{T}}_{1} \times \widetilde{\mathbf{T}}_{2}: x_{0} \leq s \leq x, y_{0} \leq t \leq y\right\}, E=\{(x, y, s, t) \in$ $\left.\widetilde{T}_{1} \times \widetilde{\mathbf{T}}_{2} \times I_{1} \times I_{2}\right\}$. $\mathcal{R}$ denotes the set of all regressive and rd-continuous functions, $\mathcal{R}^{+}=$ $\{P \in \mathcal{R}, 1+\mu(t) P(t)>0, t \in \mathbf{T}\}$. $\mathbf{R}$ denotes the set of real numbers, $\mathbf{R}_{+}=[0, \infty)$, while $\mathbf{Z}$ denotes the set of integers.

Lemma 2.1 ([33]) Let $m>0, n>0, p>0, \alpha>0$ and $\beta>0$ be given, then for each $x \geq 0$,

$$
m x^{\alpha}-n x^{\beta} \leq \frac{m(\beta-\alpha)}{\beta-p}\left(\frac{(\beta-p) n}{(\alpha-p) m}\right)^{(\alpha-p) /(\alpha-\beta)} x^{p}
$$

holds for the cases when $0<p<\alpha<\beta$ or $0<\beta<\alpha<p$.

Lemma 2.2 ([47]) Assume that $x \geq 0, p \geq q \geq 0$, and $p \neq 0$, then for any $K>0$,

$$
x^{q / p} \leq \frac{q}{p} K^{(q-p) / p} x+\frac{p-q}{p} K^{q / p} .
$$


Lemma 2.3 ([2, Theorem 6.1]) Suppose $y$ and $f$ are $r d$-continuous functions and $p \in \mathcal{R}^{+}$. Then

$$
y^{\Delta}(t) \leq p(t) y(t)+f(t), \quad \text { for all } t \in \mathbf{T}
$$

implies

$$
y(t) \leq y\left(t_{0}\right) e_{p}\left(t, t_{0}\right)+\int_{t_{0}}^{t} e_{p}(t, \sigma(\tau)) f(\tau) \Delta \tau, \quad \text { for all } t \in \mathbf{T} .
$$

Lemma 2.4 ([36]) Let $u, a, f \in C\left(\widetilde{\mathbf{T}}_{1} \times \widetilde{\mathbf{T}}_{2}, \mathbf{R}_{+}\right)$, with a and $f$ nondecreasing in each of the variables and $g \in C\left(D, \mathbf{R}_{+}\right)$be nondecreasing in $x$ and $y$. If

$$
u(x, y) \leq a(x, y)+f(x, y) \int_{x_{0}}^{x} \int_{y_{0}}^{y} g(x, y, s, t) u(s, t) \Delta t \Delta s, \quad(x, y) \in \widetilde{\mathbf{T}}_{1} \times \widetilde{\mathbf{T}}_{2},
$$

then

$$
u(x, y) \leq a(x, y) e_{p(x, y, \cdot)}\left(x, x_{0}\right), \quad(x, y) \in \widetilde{\mathbf{T}}_{1} \times \widetilde{\mathbf{T}}_{2},
$$

where $p(x, y, s)=\int_{y_{0}}^{y} f(x, y) g(x, y, s, t) \Delta t$.

Lemma 2.5 Let $u, c, d \in C\left(\widetilde{\mathbf{T}}_{1} \times \widetilde{\mathbf{T}}_{2}, \mathbf{R}_{+}\right)$and $k \geq 0$ be a constant. If

$$
u(x, y) \leq k+\int_{x_{0}}^{x} \int_{y_{0}}^{y}[c(s, t) u(s, \sigma(t))+d(s, t) u(s, t)] \Delta t \Delta s, \quad(x, y) \in \widetilde{\mathbf{T}}_{1} \times \widetilde{\mathbf{T}}_{2}
$$

then

$$
u(x, y) \leq k e_{p(\cdot, y)}\left(x, x_{0}\right), \quad(x, y) \in \widetilde{\mathbf{T}}_{1} \times \widetilde{\mathbf{T}}_{2},
$$

where

$$
\begin{aligned}
& p(x, y)=\int_{y_{0}}^{y} h(x, t) \Delta t, \quad \text { and } \\
& h(x, y)=c(x, y)+d(x, y) .
\end{aligned}
$$

Proof For an arbitrary $\varepsilon>0$, denote

$$
z(x, y)=k+\varepsilon+\int_{x_{0}}^{x} \int_{y_{0}}^{y}[c(s, t) u(s, \sigma(t))+d(s, t) u(s, t)] \Delta t \Delta s .
$$

From the assumptions, we have $z$ is positive and nondecreasing in each of the variables. By (1) and (5), we have that

$$
u(x, y) \leq z(x, y), \quad(x, y) \in \widetilde{\mathbf{T}}_{1} \times \widetilde{\mathbf{T}}_{2} .
$$


Delta differentiating with respect to the first variable and then with respect to the second, we obtain

$$
\begin{aligned}
\frac{\partial}{\Delta_{2} y}\left(\frac{\partial z(x, y)}{\Delta_{1} x}\right) & =c(x, y) u(x, \sigma(y))+d(x, y) u(x, y) \\
& \leq c(x, y) z(x, \sigma(y))+d(x, y) z(x, y), \quad(x, y) \in \widetilde{\mathbf{T}}_{1} \times \widetilde{\mathbf{T}}_{2} .
\end{aligned}
$$

From (6), we get

$$
\begin{aligned}
\frac{z(x, y) \frac{\partial}{\Delta_{2} y}\left(\frac{\Delta z(x, y)}{\Delta_{1} x}\right)}{z(x, y) z(x, \sigma(y))} & \leq\left[c(x, y)+d(x, y) \frac{z(x, y)}{z(x, \sigma(y))}\right] \\
& \leq[c(x, y)+d(x, y)] \\
& =h(x, y),
\end{aligned}
$$

where $h(x, y)$ is defined as in (4). Hence,

$$
\frac{z(x, y) \frac{\partial}{\Delta_{2} y}\left(\frac{\partial z(x, y)}{\Delta_{1} x}\right)}{z(x, y) z(x, \sigma(y))}-\frac{\frac{\partial z(x, y)}{\Delta_{1} x} \frac{\partial z(x, y)}{\Delta_{2} y}}{z(x, y) z(x, \sigma(y))} \leq h(x, y),
$$

i.e.,

$$
\frac{\partial}{\Delta_{2} y}\left(\frac{\frac{\partial z(x, y)}{\Delta_{1} x}}{z(x, y)}\right) \leq h(x, y)
$$

Delta integrating with respect to the second variable from $y_{0}$ to $y$ and noting that $\left.\frac{\partial z(x, y)}{\Delta_{1} x}\right|_{\left(x, y_{0}\right)}=0$, we have

$$
\frac{\frac{\partial z(x, y)}{\Delta_{1} x}}{z(x, y)} \leq \int_{y_{0}}^{y} h(x, t) \Delta t
$$

that is,

$$
\frac{\partial z(x, y)}{\Delta_{1} x} \leq z(x, y) \int_{y_{0}}^{y} h(x, t) \Delta t .
$$

By (3) and (8) we get

$$
\frac{\partial z(x, y)}{\Delta_{1} x} \leq p(x, y) z(x, y) .
$$

From Lemma 2.3 and $z\left(x_{0}, y\right)=k+\varepsilon$, we obtain

$$
z(x, y) \leq(k+\varepsilon) e_{p(\cdot, y)}\left(x, x_{0}\right) .
$$

Noting that $u(x, y) \leq z(x, y)$ and $\varepsilon$ is arbitrary, it follows (2). This completes the proof. 
Lemma 2.6 Let $u, c, d \in C\left(\widetilde{\mathbf{T}}_{1} \times \widetilde{\mathbf{T}}_{2}, \mathbf{R}_{+}\right)$and $k \geq 0$ be a constant. If

$$
u(x, y) \leq k+\int_{x_{0}}^{x} \int_{y_{0}}^{y}[c(s, t) u(\sigma(s), t)+d(s, t) u(s, t)] \Delta t \Delta s, \quad(x, y) \in \widetilde{\mathbf{T}}_{1} \times \widetilde{\mathbf{T}}_{2},
$$

then

$$
u(x, y) \leq k e_{p(x,)}\left(y, y_{0}\right), \quad(x, y) \in \widetilde{\mathbf{T}}_{1} \times \widetilde{\mathbf{T}}_{2}
$$

where

$$
\begin{aligned}
& p(x, y)=\int_{x_{0}}^{x} h(s, y) \Delta s, \quad \text { and } \\
& h(x, y)=c(x, y)+d(x, y) .
\end{aligned}
$$

The proof of the Lemma is similar to the proof in Lemma 2.5, and therefore is omitted.

\section{Main results}

Theorem 3.1 Let $u, a, b, h \in C\left(\widetilde{\mathbf{T}}_{1} \times \widetilde{\mathbf{T}}_{2}, \mathbf{R}_{+}\right)$, with $b$ and $h$ nondecreasing in each variable, $c, d \in C\left(D, R_{+}\right)$and let $f, g \in C\left(E, R_{+}\right)$be nondecreasing in $x$ and $y$. Assume $p, q, r, m$ and $n$ are nonnegative constants with $p \geq q, p \geq r, p \geq m, p \geq n, p \neq 0$. Suppose that $u$ satisfies the following inequality:

$$
\begin{aligned}
& u^{p}(x, y) \leq a(x, y) \\
&+b(x, y) \int_{x_{0}}^{x} \int_{y_{0}}^{y}\left[c(x, y, s, t) u^{q}(s, t)+\int_{x_{0}}^{s} \int_{y_{0}}^{t} d(x, y, \tau, \eta) u^{r}(\tau, \eta) \Delta \eta \Delta \tau\right] \Delta t \Delta s \\
&+h(x, y) \int_{x_{0}}^{\alpha} \int_{y_{0}}^{\beta}\left[f(x, y, s, t) u^{m}(s, t)\right. \\
&\left.+\int_{x_{0}}^{s} \int_{y_{0}}^{t} g(x, y, \tau, \eta) u^{n}(\tau, \eta) \Delta \eta \Delta \tau\right] \Delta t \Delta s, \\
&(x, y) \in \widetilde{\mathbf{T}}_{1} \times \widetilde{\mathbf{T}}_{2} .
\end{aligned}
$$

If there exist positive constants $K_{1}$ and $K_{2}$ such that

$$
\begin{aligned}
\lambda:= & \int_{x_{0}}^{\alpha} \int_{y_{0}}^{\beta}\left[\frac{m}{p} K_{1}^{(m-p) / p} f(\alpha, \beta, s, t) e_{R(s, t,)}\left(s, x_{0}\right)\right. \\
& \left.+\int_{x_{0}}^{s} \int_{y_{0}}^{t} \frac{n}{p} K_{2}^{(n-p) / p} g(\alpha, \beta, \tau, \eta) e_{R(\tau, \eta, \cdot)}\left(\tau, x_{0}\right) \Delta \eta \Delta \tau\right] \Delta t \Delta s \\
< & \frac{1}{h(\alpha, \beta)},
\end{aligned}
$$

then for arbitrary positive constants $K_{3}$ and $K_{4}$,

$$
u(x, y) \leq\left[a(x, y)+\frac{A(\alpha, \beta)}{1-\lambda h(\alpha, \beta)} e_{R(x, y, \cdot)}\left(x, x_{0}\right)\right]^{1 / p}, \quad(x, y) \in \widetilde{\mathbf{T}}_{1} \times \widetilde{\mathbf{T}}_{2},
$$


where

$$
\begin{aligned}
A(x, y)= & b(x, y) \int_{x_{0}}^{x} \int_{y_{0}}^{y}\left\{c(x, y, s, t)\left[\frac{q}{p} K_{3}^{(q-p) / p} a(s, t)+\frac{p-q}{p} K_{3}^{q / p}\right]\right. \\
& \left.+\int_{x_{0}}^{s} \int_{y_{0}}^{t} d(x, y, \tau, \eta)\left[\frac{r}{p} K_{4}^{(r-p) / p} a(\tau, \eta)+\frac{p-r}{p} K_{4}^{r / p}\right] \Delta \eta \Delta \tau\right\} \Delta t \Delta s \\
& +h(x, y) \int_{x_{0}}^{\alpha} \int_{y_{0}}^{\beta}\left\{f(x, y, s, t)\left[\frac{m}{p} K_{1}^{(m-p) / p} a(s, t)+\frac{p-m}{p} K_{1}^{m / p}\right]\right. \\
& \left.+\int_{x_{0}}^{s} \int_{y_{0}}^{t} g(x, y, \tau, \eta)\left[\frac{n}{p} K_{2}^{(n-p) / p} a(\tau, \eta)+\frac{p-\eta}{p} K_{2}^{n / p}\right] \Delta \eta \Delta \tau\right\} \Delta t \Delta s, \\
R(x, y, s)= & \int_{y_{0}}^{y} b(x, y) F(x, y, s, t) \Delta t, \quad \text { and } \\
F(x, y, s, t)= & \frac{q}{p} K_{3}^{(q-p) / p} c(x, y, s, t)+\int_{x_{0}}^{s} \int_{y_{0}}^{t} \frac{r}{p} K_{4}^{(r-p) / p} d(x, y, \tau, \eta) \Delta \eta \Delta \tau .
\end{aligned}
$$

Proof Denote

$$
\begin{aligned}
& z(x, y) \\
& =b(x, y) \int_{x_{0}}^{x} \int_{y_{0}}^{y}\left[c(x, y, s, t) u^{q}(s, t)+\int_{x_{0}}^{s} \int_{y_{0}}^{t} d(x, y, \tau, \eta) u^{r}(\tau, \eta) \Delta \eta \Delta \tau\right] \Delta t \Delta s \\
& \quad+h(x, y) \int_{x_{0}}^{\alpha} \int_{y_{0}}^{\beta}\left[f(x, y, s, t) u^{m}(s, t)+\int_{x_{0}}^{s} \int_{y_{0}}^{t} g(x, y, \tau, \eta) u^{n}(\tau, \eta) \Delta \eta \Delta \tau\right] \Delta t \Delta s, \\
& (x, y) \in \widetilde{\mathbf{T}}_{1} \times \widetilde{\mathbf{T}}_{2} .
\end{aligned}
$$

Then $z$ is nondecreasing in each variable on $\widetilde{\mathbf{T}}_{1} \times \widetilde{\mathbf{T}}_{2}$. From (10) and (16), we get

$$
u(x, y) \leq[a(x, y)+z(x, y)]^{1 / p}, \quad(x, y) \in \widetilde{\mathbf{T}}_{1} \times \widetilde{\mathbf{T}}_{2} .
$$

By (16) and (17), we obtain

$$
\begin{aligned}
z(x, y) \leq & b(x, y) \int_{x_{0}}^{x} \int_{y_{0}}^{y}\left\{c(x, y, s, t)[a(s, t)+z(s, t)]^{q / p}\right. \\
& \left.+\int_{x_{0}}^{s} \int_{y_{0}}^{t} d(x, y, \tau, \eta)[a(\tau, \eta)+z(\tau, \eta)]^{r / p} \Delta \eta \Delta \tau\right\} \Delta t \Delta s \\
& +h(x, y) \int_{x_{0}}^{\alpha} \int_{y_{0}}^{\beta}\left\{f(x, y, s, t)[a(s, t)+z(s, t)]^{m / p}\right. \\
& \left.+\int_{x_{0}}^{s} \int_{y_{0}}^{t} g(x, y, \tau, \eta)[a(\tau, \eta)+z(\tau, \eta)]^{n / p} \Delta \eta \Delta \tau\right\} \Delta t \Delta s, \\
& (x, y) \in \widetilde{\mathbf{T}}_{1} \times \widetilde{\mathbf{T}}_{2} .
\end{aligned}
$$

Then for $K_{1}, K_{2}$ satisfying (11) and arbitrary $K_{3}, K_{4}>0$, it follows from Lemma 2.2 that

$$
[a(x, y)+z(x, y)]^{q / p} \leq \frac{q}{p} K_{3}^{(q-p) / p}[a(x, y)+z(x, y)]+\frac{p-q}{p} K_{3}^{q / p},
$$




$$
\begin{aligned}
& {[a(x, y)+z(x, y)]^{r / p} \leq \frac{r}{p} K_{4}^{(r-p) / p}[a(x, y)+z(x, y)]+\frac{p-r}{p} K_{4}^{r / p},} \\
& {[a(x, y)+z(x, y)]^{m / p} \leq \frac{m}{p} K_{1}^{(m-p) / p}[a(x, y)+z(x, y)]+\frac{p-m}{p} K_{1}^{q / p},} \\
& {[a(x, y)+z(x, y)]^{n / p} \leq \frac{n}{p} K_{2}^{(n-p) / p}[a(x, y)+z(x, y)]+\frac{p-n}{p} K_{2}^{r / p} .}
\end{aligned}
$$

According to (18)-(22), we have

$$
\begin{aligned}
& z(x, y) \\
& \leq b(x, y) \int_{x_{0}}^{x} \int_{y_{0}}^{y}\left\{c(x, y, s, t)\left[\frac{q}{p} K_{3}^{(q-p) / p}[a(s, t)+z(s, t)]+\frac{p-q}{p} K_{3}^{q / p}\right]\right. \\
& \left.+\int_{x_{0}}^{s} \int_{y_{0}}^{t} d(x, y, \tau, \eta)\left[\frac{r}{p} K_{4}^{(r-p) / p}[a(\tau, \eta)+z(\tau, \eta)]+\frac{p-r}{p} K_{4}^{r / p}\right] \Delta \eta \Delta \tau\right\} \Delta t \Delta s \\
& +h(x, y) \int_{x_{0}}^{\alpha} \int_{y_{0}}^{\beta}\left\{f(x, y, s, t)\left[\frac{m}{p} K_{1}^{(m-p) / p}[a(s, t)+z(s, t)]+\frac{p-m}{p} K_{1}^{m / p}\right]\right. \\
& \left.+\int_{x_{0}}^{s} \int_{y_{0}}^{t} g(x, y, \tau, \eta)\left[\frac{n}{p} K_{2}^{(n-p) / p}[a(\tau, \eta)+z(\tau, \eta)]+\frac{p-n}{p} K_{2}^{n / p}\right] \Delta \eta \Delta \tau\right\} \Delta t \Delta s \\
& \leq b(x, y) \int_{x_{0}}^{x} \int_{y_{0}}^{y}\left\{c(x, y, s, t)\left[\frac{q}{p} K_{3}^{(q-p) / p} a(s, t)+\frac{p-q}{p} K_{3}^{q / p}\right]\right. \\
& \left.+\int_{x_{0}}^{s} \int_{y_{0}}^{t} d(x, y, \tau, \eta)\left[\frac{r}{p} K_{4}^{(r-p) / p} a(\tau, \eta)+\frac{p-r}{p} K_{4}^{r / p}\right] \Delta \eta \Delta \tau\right\} \Delta t \Delta s \\
& +h(x, y) \int_{x_{0}}^{\alpha} \int_{y_{0}}^{\beta}\left\{f(x, y, s, t)\left[\frac{m}{p} K_{1}^{(m-p) / p} a(s, t)+\frac{p-m}{p} K_{1}^{m / p}\right]\right. \\
& \left.+\int_{x_{0}}^{s} \int_{y_{0}}^{t} g(x, y, \tau, \eta)\left[\frac{n}{p} K_{2}^{(n-p) / p} a(\tau, \eta)+\frac{p-n}{p} K_{2}^{n / p}\right] \Delta \eta \Delta \tau\right\} \Delta t \Delta s \\
& +b(x, y) \int_{x_{0}}^{x} \int_{y_{0}}^{y}\left[\frac{q}{p} K_{3}^{(q-p) / p} c(x, y, s, t) z(s, t)\right. \\
& \left.+\int_{x_{0}}^{s} \int_{y_{0}}^{t} \frac{r}{p} K_{4}^{(r-p) / p} d(x, y, \tau, \eta) z(\tau, \eta) \Delta \eta \Delta \tau\right] \Delta t \Delta s \\
& +h(x, y) \int_{x_{0}}^{\alpha} \int_{y_{0}}^{\beta}\left[\frac{m}{p} K_{1}^{(m-p) / p} f(x, y, s, t) z(s, t)\right. \\
& \left.+\int_{x_{0}}^{s} \int_{y_{0}}^{t} \frac{n}{p} K_{2}^{(n-p) / p} g(x, y, \tau, \eta) z(\tau, \eta) \Delta \eta \Delta \tau\right] \Delta t \Delta s \\
& =A(x, y)+B(x, y)+b(x, y) \int_{x_{0}}^{x} \int_{y_{0}}^{y}\left[\frac{q}{p} K_{3}^{(q-p) / p} c(x, y, s, t) z(s, t)\right. \\
& \left.+\int_{x_{0}}^{s} \int_{y_{0}}^{t} \frac{r}{p} K_{4}^{(r-p) / p} d(x, y, \tau, \eta) z(\tau, \eta) \Delta \eta \Delta \tau\right] \Delta t \Delta s \\
& \leq A(x, y)+B(x, y)+b(x, y) \int_{x_{0}}^{x} \int_{y_{0}}^{y}\left[\frac{q}{p} K_{1}^{(q-p) / p} c(x, y, s, t) z(s, t)\right. \\
& \left.+z(s, t) \int_{x_{0}}^{s} \int_{y_{0}}^{t} \frac{r}{p} K_{4}^{(r-p) / p} d(x, y, \tau, \eta) \Delta \eta \Delta \tau\right] \Delta t \Delta s
\end{aligned}
$$




$$
\begin{aligned}
& =A(x, y)+B(x, y)+b(x, y) \int_{x_{0}}^{x} \int_{y_{0}}^{y} F(x, y, s, t) z(s, t) \Delta t \Delta s, \\
& (x, y) \in \widetilde{\mathbf{T}}_{1} \times \widetilde{\mathbf{T}}_{2},
\end{aligned}
$$

where $A(x, y)$ is defined in (13), and

$$
\begin{aligned}
B(x, y)= & h(x, y) \int_{x_{0}}^{\alpha} \int_{y_{0}}^{\beta}\left[\frac{m}{p} K_{1}^{(m-p) / p} f(x, y, s, t) z(s, t)\right. \\
& \left.+\int_{x_{0}}^{s} \int_{y_{0}}^{t} \frac{n}{p} K_{2}^{(n-p) / p} g(x, y, \tau, \eta) z(\tau, \eta) \Delta \eta \Delta \tau\right] \Delta t \Delta s .
\end{aligned}
$$

From (23) and Lemma 2.4, we have

$$
z(x, y) \leq(A(x, y)+B(x, y)) e_{R(x, y, \cdot)}\left(x, x_{0}\right), \quad(x, y) \in \widetilde{\mathbf{T}}_{1} \times \widetilde{\mathbf{T}}_{2}
$$

where $R(x, y)$ is defined in (14). By (24) and since $A, B$ are nondecreasing in each variable on $\widetilde{\mathbf{T}}_{1} \times \widetilde{\mathbf{T}}_{2}$, we obtain

$$
\begin{aligned}
z(x, y) & \leq(A(x, y)+B(x, y)) e_{R(x, y, \cdot)}\left(x, x_{0}\right) \\
& \leq(A(\alpha, \beta)+B(\alpha, \beta)) e_{R(x, y, \cdot)}\left(x, x_{0}\right) \\
& =C(\alpha, \beta) e_{R(x, y, \cdot)}\left(x, x_{0}\right)
\end{aligned}
$$

where $C(x, y)=A(x, y)+B(x, y)$. From the definitions of $B, C, \lambda$ and (25), we obtain

$$
\begin{aligned}
C(\alpha, \beta)= & A(\alpha, \beta)+B(\alpha, \beta) \\
= & A(\alpha, \beta)+h(\alpha, \beta) \int_{x_{0}}^{\alpha} \int_{y_{0}}^{\beta}\left[\frac{m}{p} K_{1}^{(m-p) / p} f(\alpha, \beta, s, t) z(s, t)\right. \\
& \left.+\int_{x_{0}}^{s} \int_{y_{0}}^{t} \frac{n}{p} K_{2}^{(n-p) / p} g(\alpha, \beta, \tau, \eta) z(\tau, \eta) \Delta \eta \Delta \tau\right] \Delta t \Delta s \\
\leq & A(\alpha, \beta)+h(\alpha, \beta) \int_{x_{0}}^{\alpha} \int_{y_{0}}^{\beta}\left[\frac{m}{p} K_{1}^{(m-p) / p} f(\alpha, \beta, s, t) C(\alpha, \beta) e_{R(s, t, \cdot)}\left(s, x_{0}\right)\right. \\
& \left.+\int_{x_{0}}^{s} \int_{y_{0}}^{t} \frac{n}{p} K_{2}^{(n-p) / p} g(\alpha, \beta, \tau, \eta) C(\alpha, \beta) e_{R(\tau, \eta, \cdot)}\left(\tau, x_{0}\right) \Delta \eta \Delta \tau\right] \Delta t \Delta s \\
= & A(\alpha, \beta)+C(\alpha, \beta) h(\alpha, \beta) \int_{x_{0}}^{\alpha} \int_{y_{0}}^{\beta}\left[\frac{m}{p} K_{1}^{(m-p) / p} f(\alpha, \beta, s, t) e_{R(s, t, \cdot)}\left(s, x_{0}\right)\right. \\
& \left.+\int_{x_{0}}^{s} \int_{y_{0}}^{t} \frac{n}{p} K_{2}^{(n-p) / p} g(\alpha, \beta, \tau, \eta) e_{R(\tau, \eta, \cdot)}\left(\tau, x_{0}\right) \Delta \eta \Delta \tau\right] \Delta t \Delta s \\
= & A(\alpha, \beta)+\lambda C(\alpha, \beta) h(\alpha, \beta) .
\end{aligned}
$$

So we get

$$
C(\alpha, \beta) \leq \frac{A(\alpha, \beta)}{1-\lambda h(\alpha, \beta)}
$$

Noting (17), (25), and (26), we get the desired inequality (12). This completes the proof. 
Remark 3.1 If we take $\mathbf{T}=\mathbf{N}, b(x, y)=h(x, y) \equiv 1$, then Theorem 3.1 reduces to [38, Theorem 5]. If we take $\mathbf{T}=\mathbf{N}, b(x, y)=h(x, y) \equiv 1, c(x, y, s, t)=c(s, t), f(x, y, s, t)=c(s, t)$, $d(x, y, s, t)=g(x, y, s, t) \equiv 0$, then Theorem 3.1 reduces to [39, Theorem 2.1].

Theorem 3.2 Assume $l \in C\left(\widetilde{\mathbf{T}}_{1} \times \widetilde{\mathbf{T}}_{2}, \mathbf{R}_{+}\right)$and $b \in C\left(\widetilde{\mathbf{T}}_{1} \times \widetilde{\mathbf{T}}_{2},(0, \infty)\right)$ are nondecreasing in each variable, $v \in C(D,(0, \infty))$ is nondecreasing in $x$ and $y, w \in C(D,(0, \infty))$ is nonincreasing in $x$ and $y$. Assume $u, a, c, d, f, g, h, p, q, r, m$ and $n$ are defined as in Theorem 3.1; while $k$ and $\theta$ are nonnegative constants with $0<p<k<\theta$ or $0<\theta<k<p$. Suppose that $u$ satisfies the following inequality:

$$
\begin{aligned}
& u^{p}(x, y) \\
& \leq a(x, y) \\
& \quad+b(x, y) \int_{x_{0}}^{x} \int_{y_{0}}^{y}\left[c(x, y, s, t) u^{q}(s, t)+\int_{x_{0}}^{s} \int_{y_{0}}^{t} d(x, y, \tau, \eta) u^{r}(\tau, \eta) \Delta \eta \Delta \tau\right] \Delta t \Delta s \\
& \quad+l(x, y) \int_{x_{0}}^{x} \int_{y_{0}}^{y}\left[v(x, y, s, t) u^{k}(s, t)-w(x, y, s, t) u^{\theta}(s, t)\right] \Delta t \Delta s \\
& \quad+h(x, y) \int_{x_{0}}^{\alpha} \int_{y_{0}}^{\beta}\left[f(x, y, s, t) u^{m}(s, t)+\int_{x_{0}}^{s} \int_{y_{0}}^{t} g(x, y, \tau, \eta) u^{n}(\tau, \eta) \Delta \eta \Delta \tau\right] \Delta t \Delta s, \\
& (x, y) \in \widetilde{\mathbf{T}}_{1} \times \widetilde{\mathbf{T}}_{2} .
\end{aligned}
$$

If there exist positive constants $K_{1}$ and $K_{2}$ such that

$$
\begin{aligned}
\tilde{\lambda}:= & \int_{x_{0}}^{\alpha} \int_{y_{0}}^{\beta}\left[\frac{m}{p} K_{1}^{(m-p) / p} f(\alpha, \beta, s, t) e_{\widetilde{R}(s, t, \cdot)}\left(s, x_{0}\right)\right. \\
& \left.+\int_{x_{0}}^{s} \int_{y_{0}}^{t} \frac{n}{p} K_{2}^{(n-p) / p} g(\alpha, \beta, \tau, \eta) e_{\widetilde{R}(\tau, \eta, \cdot)}\left(\tau, x_{0}\right) \Delta \eta \Delta \tau\right] \Delta t \Delta s \\
< & \frac{1}{h(\alpha, \beta)},
\end{aligned}
$$

then for arbitrary positive constants $K_{3}$ and $K_{4}$,

$$
u(x, y) \leq\left[a(x, y)+\frac{\widetilde{A}(\alpha, \beta)}{1-\widetilde{\lambda} h(\alpha, \beta)} e_{\widetilde{R}(x, y, \cdot)}\left(x, x_{0}\right)\right]^{1 / p}, \quad(x, y) \in \widetilde{\mathbf{T}}_{1} \times \widetilde{\mathbf{T}}_{2},
$$

where

$$
\begin{aligned}
\widetilde{A}(x, y)= & b(x, y) \int_{x_{0}}^{x} \int_{y_{0}}^{y}\left\{c(x, y, s, t)\left[\frac{q}{p} K_{3}^{(q-p) / p} a(s, t)+\frac{p-q}{p} K_{3}^{q / p}\right]\right. \\
& \left.+\int_{x_{0}}^{s} \int_{y_{0}}^{t} d(x, y, \tau, \eta)\left[\frac{r}{p} K_{4}^{(r-p) / p} a(\tau, \eta)+\frac{p-r}{p} K_{4}^{r / p}\right] \Delta \eta \Delta \tau\right\} \Delta t \Delta s \\
& +l(x, y) \int_{x_{0}}^{x} \int_{y_{0}}^{y} \varphi(x, y, s, t) a(s, t) \Delta t \Delta s \\
& +h(x, y) \int_{x_{0}}^{\alpha} \int_{y_{0}}^{\beta}\left\{f(x, y, s, t)\left[\frac{m}{p} K_{1}^{(m-p) / p} a(s, t)+\frac{p-m}{p} K_{1}^{m / p}\right]\right.
\end{aligned}
$$




$$
\begin{aligned}
& \left.+\int_{x_{0}}^{s} \int_{y_{0}}^{t} g(x, y, \tau, \eta)\left[\frac{n}{p} K_{2}^{(n-p) / p} a(\tau, \eta)+\frac{p-n}{p} K_{2}^{n / p}\right] \Delta \eta \Delta \tau\right\} \Delta t \Delta s, \\
\widetilde{R}(x, y, s)= & \int_{y_{0}}^{y} b(x, y) \widetilde{F}(x, y, s, t) \Delta t, \\
\widetilde{F}(x, y, s, t)= & \frac{q}{p} K_{3}^{(q-p) / p} c(x, y, s, t)+\frac{l(x, y)}{b(x, y)} \varphi(x, y, s, t) \\
& +\int_{x_{0}}^{s} \int_{y_{0}}^{t} \frac{r}{p} K_{4}^{(r-p) / p} d(x, y, \tau, \eta) \Delta \eta \Delta \tau, \quad \text { and } \\
\varphi(x, y, s, t)= & \frac{v(x, y, s, t)(\theta-k)}{\theta-p}\left(\frac{(\theta-p) w(x, y, s, t)}{(k-p) v(x, y, s, t)}\right)^{(k-p) /(k-\theta)} .
\end{aligned}
$$

Proof From Lemma 2.1 and (27), we have

$$
\begin{aligned}
& u^{p}(x, y) \\
& \leq a(x, y) \\
& \quad+b(x, y) \int_{x_{0}}^{x} \int_{y_{0}}^{y}\left[c(x, y, s, t) u^{q}(s, t)+\int_{x_{0}}^{s} \int_{y_{0}}^{t} d(x, y, \tau, \eta) u^{r}(\tau, \eta) \Delta \eta \Delta \tau\right] \Delta t \Delta s \\
& \quad+l(x, y) \int_{x_{0}}^{x} \int_{y_{0}}^{y} \varphi(x, y, s, t) u^{p}(s, t) \Delta t \Delta s \\
& \quad+h(x, y) \int_{x_{0}}^{\alpha} \int_{y_{0}}^{\beta}\left[f(x, y, s, t) u^{m}(s, t)+\int_{x_{0}}^{s} \int_{y_{0}}^{t} g(x, y, \tau, \eta) u^{n}(\tau, \eta) \Delta \eta \Delta \tau\right] \Delta t \Delta s, \\
& (x, y) \in \widetilde{\mathbf{T}}_{1} \times \widetilde{\mathbf{T}}_{2} .
\end{aligned}
$$

Denote

$$
\begin{aligned}
& z(x, y) \\
& =b(x, y) \int_{x_{0}}^{x} \int_{y_{0}}^{y}\left[c(x, y, s, t) u^{q}(s, t)+\int_{x_{0}}^{s} \int_{y_{0}}^{t} d(x, y, \tau, \eta) u^{r}(\tau, \eta) \Delta \eta \Delta \tau\right] \Delta t \Delta s \\
& \quad+l(x, y) \int_{x_{0}}^{x} \int_{y_{0}}^{y} \varphi(x, y, s, t) u^{p}(s, t) \Delta t \Delta s \\
& \quad+h(x, y) \int_{x_{0}}^{\alpha} \int_{y_{0}}^{\beta}\left[f(x, y, s, t) u^{m}(s, t)+\int_{x_{0}}^{s} \int_{y_{0}}^{t} g(x, y, \tau, \eta) u^{n}(\tau, \eta) \Delta \eta \Delta \tau\right] \Delta t \Delta s, \\
& (x, y) \in \widetilde{\mathbf{T}}_{1} \times \widetilde{\mathbf{T}}_{2} .
\end{aligned}
$$

From the assumptions on $v$ and $w$, we have that $\varphi$ is nondecreasing in $x$ and $y$, then $z$ is nondecreasing in each variable on $\widetilde{\mathbf{T}}_{1} \times \widetilde{\mathbf{T}}_{2}$. From (34) and (35), we get

$$
u(x, y) \leq[a(x, y)+z(x, y)]^{1 / p}, \quad(x, y) \in \widetilde{\mathbf{T}}_{1} \times \widetilde{\mathbf{T}}_{2}
$$


By (35) and (36), we obtain

$$
\begin{aligned}
& z(x, y) \leq b(x, y) \int_{x_{0}}^{x} \int_{y_{0}}^{y}\left\{c(x, y, s, t)[a(s, t)+z(s, t)]^{q / p}\right. \\
&\left.+\int_{x_{0}}^{s} \int_{y_{0}}^{t} d(x, y, \tau, \eta)[a(\tau, \eta)+z(\tau, \eta)]^{r / p} \Delta \eta \Delta \tau\right\} \Delta t \Delta s \\
&+l(x, y) \int_{x_{0}}^{x} \int_{y_{0}}^{y} \varphi(x, y, s, t)[a(s, t)+z(s, t)] \Delta t \Delta s \\
&+h(x, y) \int_{x_{0}}^{\alpha} \int_{y_{0}}^{\beta}\left\{f(x, y, s, t)[a(s, t)+z(s, t)]^{m / p}\right. \\
&\left.+\int_{x_{0}}^{s} \int_{y_{0}}^{t} g(x, y, \tau, \eta)[a(\tau, \eta)+z(\tau, \eta)]^{n / p} \Delta \eta \Delta \tau\right\} \Delta t \Delta s, \\
&(x, y) \in \widetilde{\mathbf{T}}_{1} \times \widetilde{\mathbf{T}}_{2} .
\end{aligned}
$$

For $K_{1}, K_{2}$ satisfying (28) and arbitrary $K_{3}, K_{4}>0$, it follows from Lemma 2.2 that

$$
\begin{aligned}
& {[a(x, y)+z(x, y)]^{q / p} \leq \frac{q}{p} K_{3}^{(q-p) / p}[a(x, y)+z(x, y)]+\frac{p-q}{p} K_{3}^{q / p},} \\
& {[a(x, y)+z(x, y)]^{r / p} \leq \frac{r}{p} K_{4}^{(r-p) / p}[a(x, y)+z(x, y)]+\frac{p-r}{p} K_{4}^{r / p},} \\
& {[a(x, y)+z(x, y)]^{m / p} \leq \frac{m}{p} K_{1}^{(m-p) / p}[a(x, y)+z(x, y)]+\frac{p-m}{p} K_{1}^{q / p},} \\
& {[a(x, y)+z(x, y)]^{n / p} \leq \frac{n}{p} K_{2}^{(n-p) / p}[a(x, y)+z(x, y)]+\frac{p-n}{p} K_{2}^{r / p} .}
\end{aligned}
$$

According to (37)-(41), we have

$$
\begin{aligned}
z(x, y) & \\
\leq & b(x, y) \int_{x_{0}}^{x} \int_{y_{0}}^{y}\left\{c(x, y, s, t)\left[\frac{q}{p} K_{3}^{(q-p) / p}[a(s, t)+z(s, t)]+\frac{p-q}{p} K_{3}^{q / p}\right]\right. \\
& \left.+\int_{x_{0}}^{s} \int_{y_{0}}^{t} d(x, y, \tau, \eta)\left[\frac{r}{p} K_{4}^{(r-p) / p}[a(\tau, \eta)+z(\tau, \eta)]+\frac{p-r}{p} K_{4}^{r / p}\right] \Delta \eta \Delta \tau\right\} \Delta t \Delta s \\
& +l(x, y) \int_{x_{0}}^{x} \int_{y_{0}}^{y} \varphi(x, y, s, t)[a(s, t)+z(s, t)] \Delta t \Delta s \\
& +h(x, y) \int_{x_{0}}^{\alpha} \int_{y_{0}}^{\beta}\left\{f(x, y, s, t)\left[\frac{m}{p} K_{1}^{(m-p) / p}[a(s, t)+z(s, t)]+\frac{p-m}{p} K_{1}^{m / p}\right]\right. \\
& \left.+\int_{x_{0}}^{s} \int_{y_{0}}^{t} g(x, y, \tau, \eta)\left[\frac{n}{p} K_{2}^{(n-p) / p}[a(\tau, \eta)+z(\tau, \eta)]+\frac{p-n}{p} K_{2}^{n / p}\right] \Delta \eta \Delta \tau\right\} \Delta t \Delta s \\
\leq & b(x, y) \int_{x_{0}}^{x} \int_{y_{0}}^{y}\left\{c(x, y, s, t)\left[\frac{q}{p} K_{3}^{(q-p) / p} a(s, t)+\frac{p-q}{p} K_{3}^{q / p}\right]\right. \\
& \left.+\int_{x_{0}}^{s} \int_{y_{0}}^{t} d(x, y, \tau, \eta)\left[\frac{r}{p} K_{4}^{(r-p) / p} a(\tau, \eta)+\frac{p-r}{p} K_{4}^{r / p}\right] \Delta \eta \Delta \tau\right\} \Delta t \Delta s \\
+ & l(x, y) \int_{x_{0}}^{x} \int_{y_{0}}^{y} \varphi(x, y, s, t) a(s, t) \Delta t \Delta s
\end{aligned}
$$




$$
\begin{aligned}
& +h(x, y) \int_{x_{0}}^{\alpha} \int_{y_{0}}^{\beta}\left\{f(x, y, s, t)\left[\frac{m}{p} K_{1}^{(m-p) / p} a(s, t)+\frac{p-m}{p} K_{1}^{m / p}\right]\right. \\
& \left.+\int_{x_{0}}^{s} \int_{y_{0}}^{t} g(x, y, \tau, \eta)\left[\frac{n}{p} K_{2}^{(n-p) / p} a(\tau, \eta)+\frac{p-\eta}{p} K_{2}^{n / p}\right] \Delta \eta \Delta \tau\right\} \Delta t \Delta s \\
& +b(x, y) \int_{x_{0}}^{x} \int_{y_{0}}^{y}\left[\frac{q}{p} K_{3}^{(q-p) / p} c(x, y, s, t) z(s, t)\right. \\
& \left.+\int_{x_{0}}^{s} \int_{y_{0}}^{t} \frac{r}{p} K_{4}^{(r-p) / p} d(x, y, \tau, \eta) z(\tau, \eta) \Delta \eta \Delta \tau\right] \Delta t \Delta s \\
& +l(x, y) \int_{x_{0}}^{x} \int_{y_{0}}^{y} \varphi(x, y, s, t) z(s, t) \Delta t \Delta s \\
& +h(x, y) \int_{x_{0}}^{\alpha} \int_{y_{0}}^{\beta}\left[\frac{m}{p} K_{1}^{(m-p) / p} f(x, y, s, t) z(s, t)\right. \\
& \left.+\int_{x_{0}}^{s} \int_{y_{0}}^{t} \frac{n}{p} K_{2}^{(n-p) / p} g(x, y, \tau, \eta) z(\tau, \eta) \Delta \eta \Delta \tau\right] \Delta t \Delta s \\
= & \widetilde{A}(x, y)+B(x, y)+b(x, y) \int_{x_{0}}^{x} \int_{y_{0}}^{y}\left[\frac{q}{p} K_{3}^{(q-p) / p} c(x, y, s, t) z(s, t)\right. \\
= & \widetilde{A}(x, y)+B(x, y)+b(x, y) \int_{x_{0}}^{x} \int_{y_{0}}^{y} \widetilde{F}(x, y, s, t) z(s, t) \Delta t \Delta s, \\
\leq & \widetilde{A}(x, y)+B(x, y)+b(x, y) \int_{x_{0}}^{x} \int_{y_{0}}^{y}\left[\frac{q}{p} K_{3}^{(q-p) / p} c(x, y, s, t)+\frac{l(x, y)}{b(x, y)} \varphi(x, y, s, t)\right. \\
& \left.+\int_{x_{0}}^{s} \int_{y_{0}}^{t} \frac{r}{p} K_{4}^{(r-p) / p} d(x, y, \tau, \eta) z(\tau, \eta) \Delta \eta \Delta \tau\right] \Delta t \Delta s \\
& +l(x, y) \int_{x_{0}}^{x} \int_{y_{0}}^{y} \varphi(x, y, s, t) z(s, t) \Delta t \Delta s \\
= & \widetilde{A}(x, y)+B(x, y)+b(x, y) \int_{x_{0}}^{x} \int_{y_{0}}^{y}\left[\frac{q}{p} K_{3}^{(q-p) / p) / p} c(x, y, s, t) z(s, t)\right. \\
& \left.+\int_{x_{0}}^{s} \int_{y_{0}}^{t} \frac{r}{p} K_{4}^{(r-p) / p} d(x, y, \tau, \eta) z(\tau, \eta) \Delta \eta \Delta \tau\right] \Delta t \Delta s \\
+ & (x, y) \int_{x_{0}}^{x} \int_{y_{0}}^{y} \frac{l(x, y)}{b(x, y)} \varphi(x, y, s, t) z(s, t) \Delta t \Delta s \\
& \\
& \\
&
\end{aligned}
$$

where $\widetilde{A}$ and $\widetilde{F}$ are defined in (30) and (32),

$$
\begin{aligned}
B(x, y)= & h(x, y) \int_{x_{0}}^{\alpha} \int_{y_{0}}^{\beta}\left[\frac{m}{p} K_{1}^{(m-p) / p} f(x, y, s, t) z(s, t)\right. \\
& \left.+\int_{x_{0}}^{s} \int_{y_{0}}^{t} \frac{n}{p} K_{2}^{(n-p) / p} g(x, y, \tau, \eta) z(\tau, \eta) \Delta \eta \Delta \tau\right] \Delta t \Delta s .
\end{aligned}
$$

The rest of the argument is similar to that of Theorem 3.1, and therefore is omitted. This completes the proof. 
Theorem 3.3 Assume that $u, a \in C\left(\widetilde{\mathbf{T}}_{1} \times \widetilde{\mathbf{T}}_{2}, \mathbf{R}_{+}\right)$, while $b, c, d, f, g, h, p, q, r, m$ and $n$ are defined as in Theorem 3.1. Suppose that $u$ satisfies the following inequality:

$$
\begin{aligned}
& u^{p}(x, y) \leq a(x, y) \\
&+b(x, y) \int_{x_{0}}^{x} \int_{y_{0}}^{y}\left[c(x, y, s, t) u^{q}(s, \sigma(t))+d(x, y, s, t) u^{r}(s, t)\right] \Delta t \Delta s \\
&+h(x, y) \int_{x_{0}}^{\alpha} \int_{y_{0}}^{\beta}\left[f(x, y, s, t) u^{m}(s, t)\right. \\
&\left.+\int_{x_{0}}^{s} \int_{y_{0}}^{t} g(x, y, \tau, \eta) u^{n}(\tau, \eta) \Delta \eta \Delta \tau\right] \Delta t \Delta s, \\
&(x, y) \in \widetilde{\mathbf{T}}_{1} \times \widetilde{\mathbf{T}}_{2} .
\end{aligned}
$$

If there exist positive constants $K_{1}$ and $K_{2}$ such that

$$
\begin{aligned}
\xi:= & \int_{x_{0}}^{\alpha} \int_{y_{0}}^{\beta}\left[\frac{m}{p} K_{1}^{(m-p) / p} f(\alpha, \beta, s, t) e_{Q(\cdot, t)}\left(s, x_{0}\right)\right. \\
& \left.+\int_{x_{0}}^{s} \int_{y_{0}}^{t} \frac{n}{p} K_{2}^{(n-p) / p} g(\alpha, \beta, \tau, \eta) e_{Q(\cdot, \eta)}\left(\tau, x_{0}\right) \Delta \eta \Delta \tau\right] \Delta t \Delta s \\
< & \frac{1}{h(\alpha, \beta)}
\end{aligned}
$$

then for arbitrary positive constants $K_{3}$ and $K_{4}$,

$$
u(x, y) \leq\left[a(x, y)+\frac{\widetilde{A}(\alpha, \beta)}{1-\xi h(\alpha, \beta)} e_{Q(\cdot, y)}\left(x, x_{0}\right)\right]^{1 / p}, \quad(x, y) \in \widetilde{\mathbf{T}}_{1} \times \widetilde{\mathbf{T}}_{2}
$$

where

$$
\begin{aligned}
\tilde{A}(x, y)= & b(x, y) \int_{x_{0}}^{x} \int_{y_{0}}^{y}\left\{c(x, y, s, t)\left[\frac{q}{p} K_{3}^{(q-p) / p} a(s, \sigma(t))+\frac{p-q}{p} K_{3}^{q / p}\right]\right. \\
& \left.+d(x, y, s, t)\left[\frac{r}{p} K_{4}^{(r-p) / p} a(s, t)+\frac{p-r}{p} K_{4}^{r / p}\right]\right\} \Delta t \Delta s \\
& +h(x, y) \int_{x_{0}}^{\alpha} \int_{y_{0}}^{\beta}\left\{f(x, y, s, t)\left[\frac{m}{p} K_{1}^{(m-p) / p} a(s, t)+\frac{p-m}{p} K_{1}^{m / p}\right]\right. \\
& +\int_{x_{0}}^{s} \int_{y_{0}}^{t} g(x, y, \tau, \eta)\left[\frac{n}{p} K_{2}^{(n-p) / p} a(\tau, \eta)\right. \\
& \left.\left.+\frac{p-n}{p} K_{2}^{n / p}\right] \Delta \eta \Delta \tau\right\} \Delta t \Delta s, \\
Q(x, y)= & \int_{y_{0}}^{y} h(\alpha, \beta, x, t) \Delta t, \quad \text { and } \\
h(\alpha, \beta, s, t)= & \frac{q}{p} K_{3}^{(q-p) / p} b(\alpha, \beta) c(\alpha, \beta, s, t)+\frac{r}{p} K_{4}^{(r-p) / p} b(\alpha, \beta) d(\alpha, \beta, s, t) .
\end{aligned}
$$




\section{Proof Denote}

$$
\begin{aligned}
& z(x, y) \\
& =b(x, y) \int_{x_{0}}^{x} \int_{y_{0}}^{y}\left[c(x, y, s, t) u^{q}(s, \sigma(t))+d(x, y, s, t) u^{r}(s, t)\right] \Delta t \Delta s \\
& \quad+h(x, y) \int_{x_{0}}^{\alpha} \int_{y_{0}}^{\beta}\left[f(x, y, s, t) u^{m}(s, t)\right. \\
& \left.+\int_{x_{0}}^{s} \int_{y_{0}}^{t} g(x, y, \tau, \eta) u^{n}(\tau, \eta) \Delta \eta \Delta \tau\right] \Delta t \Delta s .
\end{aligned}
$$

Then $z$ is nondecreasing in each variable on $\widetilde{\mathbf{T}}_{1} \times \widetilde{\mathbf{T}}_{2}$. From (42) and (48), we get

$$
u(x, y) \leq[a(x, y)+z(x, y)]^{1 / p}, \quad(x, y) \in \widetilde{\mathbf{T}}_{1} \times \widetilde{\mathbf{T}}_{2}
$$

By (48) and (49), we obtain

$$
\begin{aligned}
& z(x, y) \leq b(x, y) \int_{x_{0}}^{x} \int_{y_{0}}^{y}\left\{c(x, y, s, t)[a(s, \sigma(t))+z(s, \sigma(t))]^{q / p}\right. \\
&\left.+d(x, y, s, t)[a(s, t)+z(s, t)]^{r / p} \Delta \eta \Delta \tau\right\} \Delta t \Delta s \\
&+h(x, y) \int_{x_{0}}^{\alpha} \int_{y_{0}}^{\beta}\left\{f(x, y, s, t)[a(s, t)+z(s, t)]^{m / p}\right. \\
&\left.+\int_{x_{0}}^{s} \int_{y_{0}}^{t} g(x, y, \tau, \eta)[a(\tau, \eta)+z(\tau, \eta)]^{n / p} \Delta \eta \Delta \tau\right\} \Delta t \Delta s, \\
&(x, y) \in \widetilde{\mathbf{T}}_{1} \times \widetilde{\mathbf{T}}_{2} .
\end{aligned}
$$

For $K_{1}, K_{2}$ satisfying (43) and arbitrary $K_{3}, K_{4}>0$, it follows from Lemma 2.2 that

$$
\begin{aligned}
& {[a(x, \sigma(y))+z(x, \sigma(y))]^{q / p} \leq \frac{q}{p} K_{3}^{(q-p) / p}[a(x, \sigma(y))+z(x, \sigma(y))]+\frac{p-q}{p} K_{3}^{q / p},} \\
& {[a(x, y)+z(x, y)]^{r / p} \leq \frac{r}{p} K_{4}^{(r-p) / p}[a(x, y)+z(x, y)]+\frac{p-r}{p} K_{4}^{r / p},} \\
& {[a(x, y)+z(x, y)]^{m / p} \leq \frac{m}{p} K_{1}^{(m-p) / p}[a(x, y)+z(x, y)]+\frac{p-m}{p} K_{1}^{m / p},} \\
& {[a(x, y)+z(x, y)]^{n / p} \leq \frac{n}{p} K_{2}^{(n-p) / p}[a(x, y)+z(x, y)]+\frac{p-n}{p} K_{2}^{n / p} .}
\end{aligned}
$$

According to (50)-(54), we have

$$
\begin{aligned}
& z(x, y) \\
& \leq b(x, y) \int_{x_{0}}^{x} \int_{y_{0}}^{y}\left\{c ( x , y , s , t ) \left[\frac{q}{p} K_{3}^{(q-p) / p}[a(s, \sigma(t))+z(s, \sigma(t))]\right.\right. \\
& \left.\quad+\frac{p-q}{p} K_{3}^{q / p}\right]
\end{aligned}
$$




$$
\begin{aligned}
& \left.+d(x, y, s, t)\left[\frac{r}{p} K_{4}^{(r-p) / p}[a(s, t)+z(s, t)]+\frac{p-r}{p} K_{4}^{r / p}\right]\right\} \Delta t \Delta s \\
& +h(x, y) \int_{x_{0}}^{\alpha} \int_{y_{0}}^{\beta}\left\{f(x, y, s, t)\left[\frac{m}{p} K_{1}^{(m-p) / p}[a(s, t)+z(s, t)]+\frac{p-m}{p} K_{1}^{m / p}\right]\right. \\
& \left.+\int_{x_{0}}^{s} \int_{y_{0}}^{t} g(x, y, \tau, \eta)\left[\frac{n}{p} K_{2}^{(n-p) / p}[a(\tau, \eta)+z(\tau, \eta)]+\frac{p-n}{p} K_{2}^{n / p}\right] \Delta \eta \Delta \tau\right\} \Delta t \Delta s \\
& \leq b(x, y) \int_{x_{0}}^{x} \int_{y_{0}}^{y}\left\{c(x, y, s, t)\left[\frac{q}{p} K_{3}^{(q-p) / p} a(s, \sigma(t))+\frac{p-q}{p} K_{3}^{q / p}\right]\right. \\
& \left.+d(x, y, s, t)\left[\frac{r}{p} K_{4}^{(r-p) / p} a(s, t)+\frac{p-r}{p} K_{4}^{r / p}\right]\right\} \Delta t \Delta s \\
& +h(x, y) \int_{x_{0}}^{\alpha} \int_{y_{0}}^{\beta}\left\{f(x, y, s, t)\left[\frac{m}{p} K_{1}^{(m-p) / p} a(s, t)+\frac{p-m}{p} K_{1}^{m / p}\right]\right. \\
& \left.+\int_{x_{0}}^{s} \int_{y_{0}}^{t} g(x, y, \tau, \eta)\left[\frac{n}{p} K_{2}^{(n-p) / p} a(\tau, \eta)+\frac{p-n}{p} K_{2}^{n / p}\right] \Delta \eta \Delta \tau\right\} \Delta t \Delta s \\
& +b(x, y) \int_{x_{0}}^{x} \int_{y_{0}}^{y}\left[\frac{q}{p} K_{3}^{(q-p) / p} c(x, y, s, t) z(s, \sigma(t))\right. \\
& \left.+\frac{r}{p} K_{4}^{(r-p) / p} d(x, y, s, t) z(s, t)\right] \Delta t \Delta s \\
& +h(x, y) \int_{x_{0}}^{\alpha} \int_{y_{0}}^{\beta}\left[\frac{m}{p} K_{1}^{(m-p) / p} f(x, y, s, t) z(s, t)\right. \\
& \left.+\int_{x_{0}}^{s} \int_{y_{0}}^{t} \frac{n}{p} K_{2}^{(n-p) / p} g(x, y, \tau, \eta) z(\tau, \eta) \Delta \eta \Delta \tau\right] \Delta t \Delta s \\
& =\widetilde{A}(x, y)+B(x, y)+b(x, y) \int_{x_{0}}^{x} \int_{y_{0}}^{y}\left[\frac{q}{p} K_{3}^{(q-p) / p} c(x, y, s, t) z(s, \sigma(t))\right. \\
& \left.+\frac{r}{p} K_{4}^{(r-p) / p} d(x, y, s, t) z(s, t)\right] \Delta t \Delta s \\
& \leq \widetilde{A}(\alpha, \beta)+B(\alpha, \beta)+b(\alpha, \beta) \int_{x_{0}}^{x} \int_{y_{0}}^{y}\left[\frac{q}{p} K_{3}^{(q-p) / p} c(\alpha, \beta, s, t) z(s, \sigma(t))\right. \\
& \left.+\frac{r}{p} K_{4}^{(r-p) / p} d(\alpha, \beta, s, t) z(s, t)\right] \Delta t \Delta s \\
& =\widetilde{C}(\alpha, \beta)+b(\alpha, \beta) \int_{x_{0}}^{x} \int_{y_{0}}^{y}\left[\frac{q}{p} K_{3}^{(q-p) / p} c(\alpha, \beta, s, t) z(s, \sigma(t))\right. \\
& \left.+\frac{r}{p} K_{4}^{(r-p) / p} d(\alpha, \beta, s, t) z(s, t)\right] \Delta t \Delta s, \\
& (x, y) \in \widetilde{\mathbf{T}}_{1} \times \widetilde{\mathbf{T}}_{2},
\end{aligned}
$$

where $\widetilde{C}(x, y)=\widetilde{A}(x, y)+B(x, y), \widetilde{A}$ is defined in (45), and

$$
\begin{aligned}
B(x, y)= & h(x, y) \int_{x_{0}}^{\alpha} \int_{y_{0}}^{\beta}\left[\frac{m}{p} K_{1}^{(m-p) / p} f(x, y, s, t) z(s, t)\right. \\
& \left.+\int_{x_{0}}^{s} \int_{y_{0}}^{t} \frac{n}{p} K_{2}^{(n-p) / p} g(x, y, \tau, \eta) z(\tau, \eta) \Delta \eta \Delta \tau\right] \Delta t \Delta s .
\end{aligned}
$$


From Lemma 2.5, we have

$$
z(x, y) \leq \widetilde{C}(\alpha, \beta) e_{Q(\cdot, y)}\left(x, x_{0}\right), \quad(x, y) \in \widetilde{\mathbf{T}}_{1} \times \widetilde{\mathbf{T}}_{2},
$$

where $Q$ is defined in (46). The rest of the proof is similar to that of Theorem 3.1, and therefore is omitted. This completes the proof.

Theorem 3.4 Assume $u, a \in C\left(\widetilde{\mathbf{T}}_{1} \times \widetilde{\mathbf{T}}_{2}, \mathbf{R}_{+}\right)$, while $b, c, d, f, g, h, p, q, r, m$ and $n$ are defined as in Theorem 3.1. Suppose that u satisfies the following inequality:

$$
\begin{aligned}
& u^{p}(x, y) \leq a(x, y) \\
&+b(x, y) \int_{x_{0}}^{x} \int_{y_{0}}^{y}\left[c(x, y, s, t) u^{q}(\sigma(s), t)+d(x, y, s, t) u^{r}(s, t)\right] \Delta t \Delta s \\
&+h(x, y) \int_{x_{0}}^{\alpha} \int_{y_{0}}^{\beta}\left[f(x, y, s, t) u^{m}(s, t)\right. \\
&\left.+\int_{x_{0}}^{s} \int_{y_{0}}^{t} g(x, y, \tau, \eta) u^{n}(\tau, \eta) \Delta \eta \Delta \tau\right] \Delta t \Delta s, \\
&(x, y) \in \widetilde{\mathbf{T}}_{1} \times \widetilde{\mathbf{T}}_{2} .
\end{aligned}
$$

If there exist positive constants $K_{1}$ and $K_{2}$ such that

$$
\begin{aligned}
\xi:= & \int_{x_{0}}^{\alpha} \int_{y_{0}}^{\beta}\left[\frac{m}{p} K_{1}^{(m-p) / p} f(\alpha, \beta, s, t) e_{\widetilde{Q}(s, \cdot)}\left(t, y_{0}\right)\right. \\
& \left.+\int_{x_{0}}^{s} \int_{y_{0}}^{t} \frac{n}{p} K_{2}^{(n-p) / p} g(\alpha, \beta, \tau, \eta) e_{\widetilde{Q}(\tau, \cdot)}\left(\eta, y_{0}\right) \Delta \eta \Delta \tau\right] \Delta t \Delta s \\
< & \frac{1}{h(\alpha, \beta)}
\end{aligned}
$$

then for arbitrary positive constants $K_{3}$ and $K_{4}$,

$$
u(x, y) \leq\left[a(x, y)+\frac{\widetilde{F}(\alpha, \beta)}{1-\xi h(\alpha, \beta)} e_{\widetilde{Q}(x, \cdot)}\left(y, y_{0}\right)\right]^{1 / p}, \quad(x, y) \in \widetilde{\mathbf{T}}_{1} \times \widetilde{\mathbf{T}}_{2}
$$

where

$$
\begin{aligned}
\widetilde{F}(x, y)= & b(x, y) \int_{x_{0}}^{x} \int_{y_{0}}^{y}\left\{c(x, y, s, t)\left[\frac{q}{p} K_{3}^{(q-p) / p} a(\sigma(s), t)+\frac{p-q}{p} K_{3}^{q / p}\right]\right. \\
& \left.+d(x, y, s, t)\left[\frac{r}{p} K_{4}^{(r-p) / p} a(s, t)+\frac{p-r}{p} K_{4}^{r / p}\right]\right\} \Delta t \Delta s \\
& +h(x, y) \int_{x_{0}}^{\alpha} \int_{y_{0}}^{\beta}\left\{f(x, y, s, t)\left[\frac{m}{p} K_{1}^{(m-p) / p} a(s, t)+\frac{p-m}{p} K_{1}^{m / p}\right]\right. \\
& \left.+\int_{x_{0}}^{s} \int_{y_{0}}^{t} g(x, y, \tau, \eta)\left[\frac{n}{p} K_{2}^{(n-p) / p} a(\tau, \eta)+\frac{p-n}{p} K_{2}^{n / p}\right] \Delta \eta \Delta \tau\right\} \Delta t \Delta s,
\end{aligned}
$$


and

$$
\widetilde{Q}(x, y)=\int_{x_{0}}^{x} h(\alpha, \beta, s, y) \Delta s .
$$

The proof of the theorem is similar to that of Theorem 3.3, and therefore is omitted.

\section{Application}

In this section, we will present an application for our results.

Example 1 Consider the following partial dynamic equation with positive and negative coefficients:

$$
\left\{\begin{array}{l}
\frac{\partial}{\Delta_{2} y}\left(\frac{\partial u(x, y)}{\Delta_{1} x}\right) \\
=c(x, y) u^{q}(x, y)+v(x, y) u^{k}(x, y) \\
\quad-w(x, y) u^{\theta}(x, y)+\int_{x_{0}}^{x} \int_{y_{0}}^{y} d(\tau, \eta) u^{r}(\tau, \eta) \Delta \eta \Delta \tau \\
\quad+g(x, y) \int_{x_{0}}^{\alpha} \int_{y_{0}}^{\beta} f(s, t) u^{m}(s, t) \Delta t \Delta s, \quad(x, y) \in \widetilde{\mathbf{T}}_{1} \times \widetilde{\mathbf{T}}_{2}, \\
u\left(x, y_{0}\right)=\phi(x), \quad u\left(x_{0}, y\right)=\psi(y), \quad u\left(x_{0}, y_{0}\right)=u_{0},
\end{array}\right.
$$

where $u, c, d, f, g \in C\left(\widetilde{\mathbf{T}}_{1} \times \widetilde{\mathbf{T}}_{2}, \mathbf{R}_{+}\right), v, w \in C\left(\widetilde{\mathbf{T}}_{1} \times \widetilde{\mathbf{T}}_{2},(0, \infty)\right), q, r$ and $m$ are nonnegative constants with $1 \geq q, 1 \geq r, 1 \geq m$. Assume $\theta$ is a quotient of an even integer over odd integer, $k$ is a nonnegative constant with $0<1<k<\theta$ or $0<\theta<k<1$.

If there exists a positive constant $K_{1}$ such that

$$
\begin{aligned}
\tilde{\lambda} & :=\int_{x_{0}}^{\alpha} \int_{y_{0}}^{\beta}\left[m K_{1}^{m-1} f(s, t) e_{\widetilde{R}(\cdot, t)}\left(s, x_{0}\right)\right] \Delta t \Delta s \\
& <\frac{1}{h(\alpha, \beta)},
\end{aligned}
$$

then for arbitrary positive constants $K_{3}$ and $K_{4}$,

$$
u(x, y) \leq a(x, y)+\frac{\widetilde{A}(\alpha, \beta)}{1-\widetilde{\lambda} h(\alpha, \beta)} e_{\widetilde{R}(\cdot, y)}\left(x, x_{0}\right), \quad(x, y) \in \widetilde{\mathbf{T}}_{1} \times \widetilde{\mathbf{T}}_{2},
$$

where

$$
\begin{aligned}
a(x, y)= & |\phi(x)|+|\psi(y)|+\left|u_{0}\right|, \\
\widetilde{A}(x, y)= & \int_{x_{0}}^{x} \int_{y_{0}}^{y}\left\{c(s, t)\left[q K_{3}^{q-1} a(s, t)+(1-q) K_{3}^{q}\right]\right. \\
& \left.+\int_{x_{0}}^{s} \int_{y_{0}}^{t} d(\tau, \eta)\left[r K_{4}^{r-1} a(\tau, \eta)+(1-r) K_{4}^{r}\right] \Delta \eta \Delta \tau\right\} \Delta t \Delta s \\
& +\int_{x_{0}}^{x} \int_{y_{0}}^{y} \varphi(s, t) a(s, t) \Delta t \Delta s \\
& +h(x, y) \int_{x_{0}}^{\alpha} \int_{y_{0}}^{\beta} f(s, t)\left[m K_{1}^{m-1} a(s, t)+(1-m) K_{1}^{m}\right] \Delta t \Delta s, \\
h(x, y)= & \int_{x_{0}}^{x} \int_{y_{0}}^{y} g(s, t) \Delta t \Delta s,
\end{aligned}
$$




$$
\begin{aligned}
& \widetilde{R}(x, y)=\int_{y_{0}}^{y} \widetilde{F}(x, t) \Delta t, \\
& \widetilde{F}(x, y)=q K_{3}^{q-1} c(x, y)+\varphi(x, y)+\int_{x_{0}}^{x} \int_{y_{0}}^{y} r K_{4}^{r-1} d(\tau, \eta) \Delta \eta \Delta \tau, \quad \text { and } \\
& \varphi(x, y)=\frac{(\theta-k) v(x, y)}{\theta-1}\left(\frac{(\theta-1) w(x, y)}{(k-1) v(x, y)}\right)^{(k-1) /(k-\theta)} .
\end{aligned}
$$

Proof Let $u(x, y)$ be a solution of (55). Then, it satisfies the following dynamical integral equation:

$$
\begin{aligned}
u(x, y)= & \phi(x)+\psi(y)-u_{0} \\
& +\int_{x_{0}}^{x} \int_{y_{0}}^{y}\left[c(s, t) u^{q}(s, t)+\int_{x_{0}}^{s} \int_{y_{0}}^{t} d(\tau, \eta) u^{r}(\tau, \eta) \Delta \eta \Delta \tau\right] \Delta t \Delta s \\
& +\int_{x_{0}}^{x} \int_{y_{0}}^{y}\left[v(s, t) u^{k}(s, t)-w(s, t) u^{\theta}(s, t)\right] \Delta t \Delta s \\
& +\int_{x_{0}}^{x} \int_{y_{0}}^{y} g(s, t) \Delta t \Delta s \int_{x_{0}}^{\alpha} \int_{y_{0}}^{\beta} f(s, t) u^{m}(s, t) \Delta t \Delta s .
\end{aligned}
$$

Then from (58), (60), and (63), we have

$$
\begin{aligned}
|u(x, y)| \leq & a(x, y) \\
& +\int_{x_{0}}^{x} \int_{y_{0}}^{y}\left[c(s, t)|u(s, t)|^{q}+\int_{x_{0}}^{s} \int_{y_{0}}^{t} d(\tau, \eta)|u(\tau, \eta)|^{r} \Delta \eta \Delta \tau\right] \Delta t \Delta s \\
& +\int_{x_{0}}^{x} \int_{y_{0}}^{y}\left[v(s, t)|u(s, t)|^{k}-w(s, t)|u(s, t)|^{\theta}\right] \Delta t \Delta s \\
& +h(x, y) \int_{x_{0}}^{\alpha} \int_{y_{0}}^{\beta} f(s, t)|u(s, t)|^{m} \Delta t \Delta s, \quad(x, y) \in \widetilde{\mathbf{T}}_{1} \times \widetilde{\mathbf{T}}_{2} .
\end{aligned}
$$

An application of Theorem 3.2 with $p=1, b(x, y)=l(x, y) \equiv 1, c(x, y, s, t)=c(s, t)$, $d(x, y, s, t)=d(s, t), \quad v(x, y, s, t)=v(s, t), \quad w(x, y, s, t)=w(s, t), f(x, y, s, t)=f(s, t)$ and $g(x, y, s, t) \equiv 0$ yields $(57)$.

\section{Conclusions}

We have established several generalized Volterra-Fredholm-type dynamical integral inequalities in two independent variables on time scale pairs using an inequality introduced in [33]. As one can see, Theorems 3.1-3.4 generalize many known results in the literature. Theorem 3.2 can be applied to deal with the bounds of solutions of certain partial dynamic equation with positive and negative coefficients. Moreover, unlike some existing results in the literature (e.g., [28, 36, 37]), the integral inequalities considered in this paper involve the forward jump operator $\sigma(x)$ on a time scale, which results in difficulties in the estimation on the explicit bounds of the unknown function $u(x, y)$. 


\section{Funding}

This research was supported by the Natural Science Foundation of Shandong Province (China) (No. ZR2018MA018), and the National Natural Science Foundations of China (Nos. 11671227, 61873144).

\section{Competing interests}

The authors declare that there is no conflict of interests regarding the publication of this paper.

\section{Authors' contributions}

All authors contributed equally and read and approved the final version of the manuscript.

\section{Author details}

'School of Mathematical Sciences, Qufu Normal University, Qufu, China. ${ }^{2}$ School of Statistics, Qufu Normal University, Qufu, China.

\section{Publisher's Note}

Springer Nature remains neutral with regard to jurisdictional claims in published maps and institutional affiliations.

\section{Received: 4 October 2019 Accepted: 8 January 2020 Published online: 15 January 2020}

\section{References}

1. Hilger, S.: Ein Maßkettenkalkül mit Anwendung auf Zentrumsmanningfaltigkeiten. PhD thesis, Universität Würzburg (1988)

2. Bohner, M., Peterson, A.: Dynamic Equations on Time Scales: An Introduction with Applications. Birkhäuser, Boston (2001)

3. Bohner, M., Peterson, A.: Advances in Dynamic Equations on Time Scales. Birkhäuser, Boston (2003)

4. Atici, F.M., Biles, D.C., Lebedinsky, A.: An application of time scales to economics. Math. Comput. Model. 43, 718-726 (2006)

5. Liu, H.D., Meng, F.W., Liu, P.C.: Oscillation and asymptotic analysis on a new generalized Emden-Fowler equation. Appl. Math. Comput. 219(5), 2739-2748 (2012)

6. Chen, D., Kou, K.I., Xia, Y.H.: Linear quaternion-valued dynamic equations on time scales. J. Appl. Anal. Comput. 8, 172-201 (2018)

7. Tunç, E., Liu, H.D.: Oscillatory behavior for second-order damped differential equation with nonlinearities including Riemann-Stieltjes integrals. Electron. J. Differ. Equ. 2018, 54 (2018)

8. Liu, H.D., Meng, F.W.: Existence of positive periodic solutions for a predator-prey system of Holling type IV function response with mutual interference and impulsive effects. Discrete Dyn. Nat. Soc. 2015, 138984 (2015)

9. Zhao, D.L., Liu, H.D.: Coexistence in a two species chemostat model with Markov switchings. Appl. Math. Lett. 94, 266-271 (2019)

10. Erbe, L., Jia, B.G., Peterson, A.: On the asymptotic behavior of solutions of Emden-Fowler equations on time scales. Ann. Mat. Pura Appl. 191, 205-217 (2012)

11. Liu, H.D., Ma, C.Q.: Oscillation criteria for second-order neutral delay dynamic equations with nonlinearities given by Riemann-Stieltjes integrals. Abstr. Appl. Anal. 2013, Article ID 530457 (2013)

12. Federson, M., Mesquita, J.G., Slavik, A.: Measure functional differential equations and functional dynamic equations on time scales. J. Differ. Equ. 252, 3816-3847 (2012)

13. Liu, H.D., Liu, P.C.: Oscillation criteria for some new generalized Emden-Fowler dynamic equations on time scales. Abstr. Appl. Anal. 2013, Article ID 962590 (2013)

14. Zhang, B., Zhuang, J.S., Liu, H.D., Cao, J.D., Xia, Y.H.: Master-slave synchronization of a class of fractional-order Takagi-Sugeno fuzzy neural networks. Adv. Differ. Equ. 2018, 473 (2018)

15. Zhao, D.L., Yuan, S.L., Liu, H.D.: Random periodic solution for a stochastic SIS epidemic model with constant population size. Adv. Differ. Equ. 2018, 64 (2018)

16. Liu, H.D., Ma, C.Q.: Oscillation criteria of even order delay dynamic equations with nonlinearities given by Riemann-Stieltjes integrals. Abstr. Appl. Anal. 2014, Article ID 395381 (2014)

17. Liu, H.D.: Lyapunov-type inequalities for certain higher-order difference equations with mixed non-linearities. Adv. Differ. Equ. 2018, 229 (2018)

18. Xia, Y.H., Li, J.B., Wong, P.J.Y.: On the topological classification of dynamic equations on time scales. Nonlinear Anal, Real World Appl. 14(6), 2231-2248 (2013)

19. Chen, L., Huang, C.D., Liu, H.D., Xia, Y.H.: Anti-synchronization of a class of chaotic systems with application to Lorenz system: a unified analysis of the integer order and fractional order. Mathematics 7(6), 559 (2019)

20. Karpuz, B.: Volterra theory on time scales. Results Math. 65(3), 263-292 (2014)

21. Liu, H.D., Meng, F.W.: Interval oscillation criteria for second-order nonlinear forced differential equations involving variable exponent. Adv. Differ. Equ. 2016, 291 (2016)

22. Zhao, D.L., Yuan, S.L., Liu, H.D.: Stochastic dynamics of the delayed chemostat with Lévy noises. Int. J. Biomath. 12(5) 1950056 (2019)

23. Liu, H.D.: Some new integral inequalities with mixed nonlinearities for discontinuous functions. Adv. Differ. Equ. 2018, $22(2018)$

24. Slavik, A.: Averaging dynamic equations on time scales. J. Math. Anal. Appl. 388, 996-1012 (2012)

25. Meng, Z.W., Zheng, B., Wen, C.B.: Some new integral inequalities on time scales containing integration on infinite intervals. J. Inequal. Appl. 2013, 245 (2013)

26. Liu, H.D.: An improvement of the Lyapunov inequality for certain higher order differential equations. J. Inequal. Appl. 2018, 215 (2018)

27. Agarwal, R.P., Bohner, M., Peterson, A.: Inequalities on time scales: a survey. Math. Inequal. Appl. 4, 535-557 (2001) 
28. Anderson, D.R.: Nonlinear dynamic integral inequalities in two independent variables on time scale pairs. Adv. Dyn. Syst. Appl. 3, 1-13 (2008)

29. Liu, H.D., Meng, F.W.: Some new nonlinear integral inequalities with weakly singular kernel and their applications to FDEs. J. Inequal. Appl. 2015, 209 (2015)

30. Bohner, E.A., Bohner, M., Akin, F.: Pachpatte inequalities on time scale. J. Inequal. Pure Appl. Math. 6(1), Article ID 6 (2005)

31. Liu, H.D.: Some new half-linear integral inequalities on time scales and applications. Discrete Dyn. Nat. Soc. 2019, 9860302 (2019)

32. Saker, S.H.: Some nonlinear dynamic inequalities on time scales. Math. Inequal. Appl. 14, 633-645 (2011)

33. Liu, H.D., Li, C.Y., Shen, F.C.: A class of new nonlinear dynamic integral inequalities containing integration on infinite interval on time scales. Adv. Differ. Equ. 2019, 311 (2019)

34. Xia, Y.H., Chen, L., Kou, K.I.: Holder regularity of Grobman-Hartman theorem for dynamic equations on measure chains. Bull. Malays. Math. Sci. Soc. 41(3), 1153-1180 (2018)

35. Pachpatte, D.B.: Explicit estimates on integral inequalities with time scale. J. Inequal. Pure Appl. Math. 7, Article ID 143 (2006)

36. Ferreira, R.A.C., Torres, D.F.M.: Some linear and nonlinear integral inequalities on time scales in two independent variables. Nonlinear Dyn. Syst. Theory 9(2), 161-169 (2009)

37. Liu, H.D., Meng, F.W.: Nonlinear retarded integral inequalities on time scales and their applications. J. Math. Inequal. 12(1), 219-234 (2018)

38. Feng, Q.H., Meng, F.W., Fu, B.S.: Some new generalized Volterra-Fredholm type finite difference inequalities involving four iterated sums. Appl. Math. Comput. 219, 8247-8258 (2013)

39. Ma, Q.H.: Estimates on some power nonlinear Volterra-Fredholm type discrete inequalities and their applications. J. Comput. Appl. Math. 233, 2170-2180 (2010)

40. Liu, H.D.: Half-linear Volterra-Fredholm type integral inequalities on time scales and their applications. J. Appl. Anal. Comput. 10(1), 234-248 (2020)

41. Meng, F.W., Shao, J.: Some new Volterra-Fredholm type dynamic integral inequalities on time scales. Appl. Math. Comput. 223, 444-451 (2013)

42. Liu, H.D., Meng, F.W.: Some new generalized Volterra-Fredholm type discrete fractional sum inequalities and their applications. J. High Energy Phys. 2016, 213 (2016)

43. Wang, J.F., Meng, F.W., Gu, J.: Estimates on some power nonlinear Volterra-Fredholm type dynamic integral inequalities on time scales. Adv. Differ. Equ. 2017, 257 (2017)

44. Liu, H.D.: On some nonlinear retarded Volterra-Fredholm type integral inequalities on time scales and their applications. J. Inequal. Appl. 2018, 211 (2018)

45. Liu, H.D.: A class of retarded Volterra-Fredholm type integral inequalities on time scales and their applications. J. Inequal. Appl. 2017, 293 (2017)

46. Gu, J., Meng, F.W.: Some new nonlinear Volterra-Fredholm type dynamic integral inequalities on time scales. Appl. Math. Comput. 245, 235-242 (2014)

47. Jiang, F.C., Meng, F.W.: Explicit bounds on some new nonlinear integral inequalities with delay. J. Comput. Appl. Math. 205, 479-486 (2007)

\section{Submit your manuscript to a SpringerOpen ${ }^{0}$ journal and benefit from:}

- Convenient online submission

- Rigorous peer review

- Open access: articles freely available online

- High visibility within the field

- Retaining the copyright to your article

Submit your next manuscript at $\gg$ springeropen.com 